\title{
Sub-barrier and Above-barrier Electron Transport Through Multilayer Semiconductors
}

\author{
Voxob Rustamovich Rasulov, Rustam Yavkachovich Rasulov, \\ Iqboljon Mamirjonovich Eshboltayev, Ravshan Rustamovich Sultanov
}

Department of Physics, Faculty of Physics and Mathematics, Fergana State University, Fergana, Uzbekistan

Email address:

r_rasulov51@mail.ru (V. R. Rasulov)

\section{To cite this article:}

Voxob Rustamovich Rasulov, Rustam Yavkachovich Rasulov, Iqboljon Mamirjonovich Eshboltayev, Ravshan Rustamovich Sultanov. Subbarrier and Above-barrier Electron Transport Through Multilayer Semiconductors. American Journal of Physics and Applications.

Vol. 8, No. 4, 2020, pp. 56-63. doi: 10.11648/j.ajpa.20200804.12

Received: July 24, 2019; Accepted: December 13, 2019; Published: September 16, 2020

\begin{abstract}
The transparency coefficients of the semiconductor structure consisting of alternating asymmetric potential barriers and wells are calculated, where taken into account the Bastard condition. It is shown that both in the above-barrier and over barrier passage of electrons, tunneling oscillations arise. The amplitude, in this case, is determined not only by the values of the wave vectors, but from the values of the effective masses of the current carriers. This oscillation does not disappear even in symmetric structures if they have a difference in the effective masses of current carriers located in two neighboring regions. In symmetrical structures, an oscillation of the coefficient of the above-barrier passage of a particle depending on its energy should be observed without taking into account the Bastard condition. Calculations show that for equal values of the width of the well and the potential barrier, as well as jumps in the potential of the barrier or well, the amplitude of the oscillations of the coefficient of over-barrier passage of particles is greater than the coefficient of passage above the well. In the case of an asymmetric structure, these considerations remain valed, but the physical nature of the parameters, for example, the number of oscillations, reflection and transmission coefficients, strongly depends on the ratio of the effective masses of electrons in neighboring layers and from the ratio of the height of the left and right potential barrier (regarding to the well). In an asymmetric (and in a symmetric, but with different effective masses of electrons in different layers) semiconductor structure, oscillation should be observed depending on the coefficient of transmission through the potential barrier on the energy of electron. This oscillation is caused by the interference of waves going to the barrier and reflected from the potential barrier. Such an interference phenomenon in the structure does not disappear even in a symmetric structure due to the difference in the effective masses of electrons located in different regions of the structure. The electronic states of a multilayer semiconductor structure consisting of alternating potential wells and barriers are analyzed.
\end{abstract}

Keywords: Semiconductor, Multilayer Structure, Barrier, Well, Electron, Transparency Coefficient

\section{Introduction}

Multilayer compositions of chemically inhomogeneous semiconductors have acquired exceptional relevance due to the extremely widespread use of these systems in micro- or nanoelectronics and in physical research "as discussed by Shuka [1]". Such systems are the main technological composition for the element base of integrated circuits and form the basis of modern semiconductor electronics "as discussed elsewhere [2, 3]."

The progress of modern microelectronics is largely determined by the research of the properties of systems with heterogeneously distributed parameters, the development of methods for effective theoretical analysis of such systems, the development and provision of objective methods for controlling technological processes that allow the creation of semiconductor layers with desired properties "as discussed elsewhere [1-4]." In this work, we consider below the general problems of the propagation of electron waves in a medium whose properties change only along a certain direction. The approach is based on the use of the one-electron stationary Schrödinger equation to describe the processes of elastic scattering and tunneling of non-interacting spinless particles, provided that their total energy is preserved. 
The research of the electronic properties of both symmetric and asymmetric in relation to the geometric dimensions of the layers of the semiconductor structure is relevant in connection with the use of these structures in micro- or nanoelectronics and in other areas of solid state physics "as discussed elsewhere [1-6]." The dynamic conductivity $\sigma(\omega)$ or the current $\mathrm{j}(\omega)$ of the system response to an external action in a semiconductor multilayer structure were calculated "as discussed elsewhere [7-17]". The theory was created in different models using various mathematical methods for solving the complete Schrödinger equation for a system of electrons interacting with an electromagnetic field in a structure with a shaped potential barrier. In these works, the problem was solved without taking into account the Bastard condition "as discussed by Bastard [2, 3]", i.e. the difference in the effective masses of current carriers in neighboring layers of the structure is not taken into account.

Modern technology makes it possible to obtain semiconductor layers with an arbitrary profile of composition change (structure with a quantum well) to improve the characteristics of devices obtained on their basis "as discussed elsewhere [1-5]." In this case, the problem of electron states is reduced to the problem of the behavior of a particle in rectangular potential wells, between two neighboring which have a potential well, described by the relation research "as discussed by Rasulov [4]" (see Figure 1)

$$
U(x)=\left\{\begin{array}{cc}
U_{j} & \text { if } x<x_{j}, \\
U_{j+1} & \text { if } x_{j+1}<x<x_{j+2}, \\
U_{j+2} & \text { if } x_{j+2}\left\langle x<x_{j+3},\right. \\
U_{j+3} & \text { if } x_{j+3}\left\langle x<x_{j+4},\right. \\
U_{j+4} & \text { if } x>x_{j+4} \cdots
\end{array}\right.
$$

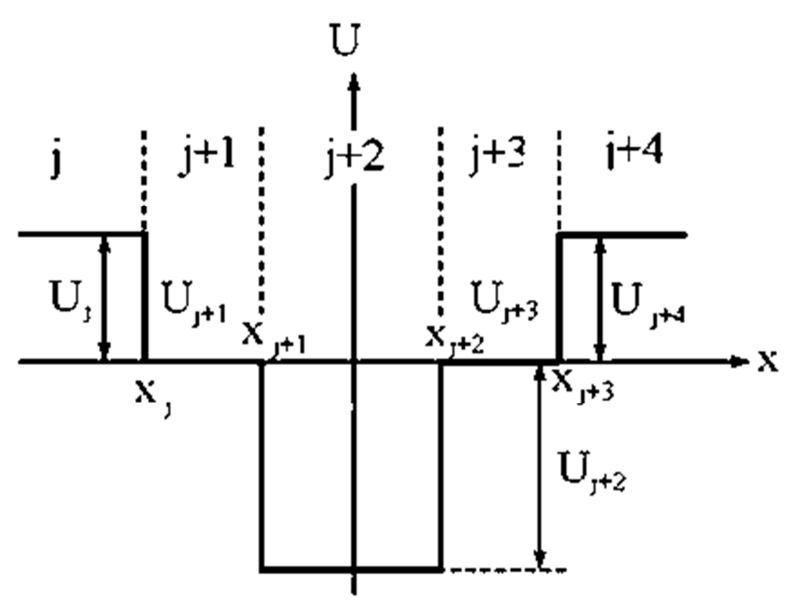

Figure 1. Structure with asymmetric potential barriers.

Further, we note that to create a new generation of resonant tunnel diodes, heterolasers with separated electronic and optical confinement, structures with rectangular sizequantized wells are used, in the center of which there is an additional energy dip. Such a structure is described by potential (1), where it is necessary to consider that $\left.U_{j}, U_{j+4}\right\rangle 0, U_{j+1}, U_{j+3}=0, U_{j+2}\langle 0$.

\section{Materials and Methods}

\subsection{General Relations}

Nanostructures grown on the basis of a narrow-gap semiconductor between two layers of wide-gap material are described as a structure with asymmetric rectangular potential barriers, i.e. with potential (1), where $U_{j}, U_{j+2}>0$, $U_{j+1}, U_{j+3}, U_{j+4}=0$ (see Figure 2).

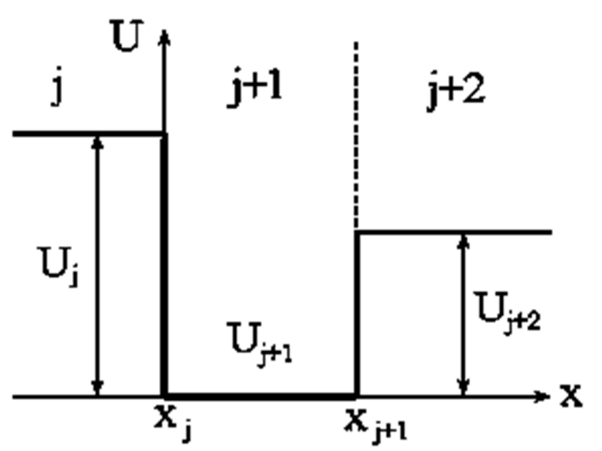

Figure 2. Asymmetric structure with a single potential well.

Then the wave function of the electron in potential (1) can be represented as

$$
\psi_{j}(x)=A_{j} e^{\left(i k_{j} x\right)}+B_{j} e^{\left(-i k_{j} x\right)}
$$

where $k_{j}(x)=k_{j}=\sqrt{\frac{2 m_{j}}{\hbar^{2}}\left(E-U_{j}\right)}, \quad j=1,2,3, \ldots$.

Further, we assume that the effective masses of the electrons in the neighboring layers are different. Therefore, the boundary conditions for the wave functions of electrons have the form "as discussed by Bastard [6]"

$$
\psi_{j}\left(x=x_{j}\right)=\psi_{j+1}\left(x=x_{j}\right),\left.\frac{1}{m_{j}} \frac{\partial \psi_{j}(x)}{\partial x}\right|_{x=x_{j}}=\left.\frac{1}{m_{j+1}} \frac{\partial \psi_{j+1}(x)}{\partial x}\right|_{x=x_{j}}
$$

Substituting (2) into (3) we obtain the expressions for the amplitudes of the electronic de Broglie waves

$$
\begin{aligned}
& 2 A_{j}=\left(1+\frac{\tilde{k}_{j+1}}{\tilde{k}_{j}}\right) A_{j+1} e^{\left(i\left(k_{j+1}-k_{j}\right) x_{j}\right)}+\left(1-\frac{\tilde{k}_{j+1}}{\tilde{k}_{j}}\right) B_{j+1} e^{\left(-i\left(k_{j+1}+k_{j}\right) x_{j}\right)}, \\
& 2 B_{j}=\left(1-\frac{\tilde{k}_{j+1}}{\tilde{k}_{j}}\right) A_{j+1} e^{\left(i\left(k_{j+1}+k_{j}\right) x_{j}\right)}+\left(1+\frac{\tilde{k}_{j+1}}{\tilde{k}_{j}}\right) B_{j+1} e^{\left(-i\left(k_{j+1}-k_{j}\right) x_{j}\right)} .
\end{aligned}
$$

where $\tilde{k}_{j}=k_{j} / m_{j}$. To simplify further calculations, we introduce the transfer matrix satisfying the following equality

$$
\left[\begin{array}{c}
A_{j} \\
B_{j}
\end{array}\right]=\hat{T}^{\left(j, j^{\prime}\right)}\left[\begin{array}{c}
A_{j^{\prime}} \\
B_{\ddot{j}}
\end{array}\right]\left[\begin{array}{ll}
T_{11}^{\left(j, j^{\prime}\right)} & T_{12}^{\left(j, j^{\prime}\right)} \\
T_{21}^{\left(j, j^{\prime}\right)} & T_{22}^{\left(j, j^{\prime}\right)}
\end{array}\right]\left[\begin{array}{c}
A_{j^{\prime}} \\
B_{\ddot{j}}
\end{array}\right],
$$

where the matrix elements in the case $j^{\prime}=j+1$ 


$$
\begin{aligned}
& T_{11}^{(j, j+1)}=\frac{1}{2}\left(1+\frac{\tilde{k}_{j+1}}{\tilde{k}_{j}}\right) e^{\left(i\left(k_{j+1}-k_{j}\right) x_{j}\right)}, T_{12}^{(j, j+1)}=\frac{1}{2}\left(1-\frac{\tilde{k}_{j+1}}{\tilde{k}_{j}}\right) e^{-i\left(k_{j+1}+k_{j}\right) x_{j}}, \\
& T_{21}^{(j, j+1)}=T_{12}^{(j, j+1) *}, T_{22}^{(j, j+1)}=T_{11}^{(j, j+1) *} .
\end{aligned}
$$

Note that for the matrix $\hat{T}^{(j, j+1)}$ we have

$$
T_{11}^{(j, j+1)}=T_{22}^{(j, j+1) *}, T_{12}^{(j, j+1)}=T_{21}^{(j, j+1) *}, T_{11}^{(j, j+1)} T_{22}^{(j, j+1)}-T_{21}^{(j, j+1)} T_{12}^{(j, j+1)}=\tilde{k}_{j+1} / \tilde{k}_{j}
$$

the matrix $\hat{T}^{(j, j+1)}$ becomes a unipolar matrix in the case $\tilde{k}_{j+1}=\tilde{k}_{j}$, i.e. for symmetric structures "as discussed by Ivchenko and Pikus [6]," when the height of potential barriers and the effective masses of electrons are the same.

We note here that a similar problem was solved in "as discussed elsewhere [7-11]." for a symmetric structure without taking the Bastard condition into account, and "as discussed elsewhere [12-18]" for structures with a potential barrier.

\subsection{Electronic Properties of the Structure with one Potential Barrier}

Now consider the concrete cases: let the three-layer structure be in the middle of one potential barrier (see Figure

3 ). Then the reflection coefficients $\left(r_{j, j+2}\right)$ of the potential barrier and the passage through the potential barrier introduced $\left(t_{j, j+2}\right)$ as ratios of the density of probability flow in the reflected and transmitted de Broglie waves of electrons in the incident wave, in the form of the transfer matrix, have view

$$
\begin{gathered}
r_{j, j+2}=1-t_{j, j+2}, \\
t_{j, j+2}=\frac{4 \frac{k_{j+2}}{k_{j}} \frac{m_{j}}{m_{j+2}}}{\left(1+\frac{k_{j+2}}{k_{j}} \frac{m_{j}}{m_{j+2}}\right)^{2}-\left[1-\left(\frac{k_{j+1}}{k_{j}} \frac{m_{j}}{m_{j+1}}\right)^{2}\left[1-\left(\frac{k_{j+2}}{k_{j+1}} \frac{m_{j+1}}{m_{j+2}}\right)^{2}\right] \sin ^{2}\left[k_{j+1}\left(x_{j+1}-x_{j}\right)\right]\right.} .
\end{gathered}
$$

Where it was consedered that electron transfer occurs according to the scheme $j \rightarrow j+1 \rightarrow j+2$. Then the matrix elements of the transfer matrix $\widehat{T}^{(j, j+2)}=\widehat{T}^{(j, j+1)} \widehat{T}^{(j+1, j+2)}$ :

$$
\begin{gathered}
T_{11}^{(j, j+2)}=T_{11}^{(j, j+1)} T_{11}^{(j+1, j+2)}+T_{12}^{(j, j+1)} T_{21}^{(j+1, j+2)}=\left(4 \tilde{k}_{j} \tilde{k}_{j+1}\right)^{-1} e^{\left(i\left(k_{j+2} x_{j+1}-k_{j} x_{j}\right)\right)} \times \\
\times\left\{\left(\tilde{k}_{j}+\tilde{k}_{j+1}\right)\left(\tilde{k}_{j+1}+\tilde{k}_{j+2}\right) e^{\left[-i k_{j+1}\left(x_{j+1}-x_{j}\right)\right]}+\left(\tilde{k}_{j}-\tilde{k}_{j+1}\right)\left(\tilde{k}_{j+1}-\tilde{k}_{j+2}\right) e^{\left[i k_{j+1}\left(x_{j+1}-x_{j}\right)\right]}\right\}, \\
T_{12}^{(j, j+2)}=T_{12}^{(j, j+1)} T_{22}^{(j+1, j+2)}+T_{11}^{(j, j+1)} T_{12}^{(j+1, j+2)}=\left(4 \tilde{k}_{j} \tilde{k}_{j+1}\right)^{-1} e^{\left(-i\left(k_{j+2} x_{j+1}-k_{j} x_{j}\right)\right)} \times \\
\times\left\{\left(\tilde{k}_{j}-\tilde{k}_{j+1}\right)\left(\tilde{k}_{j+1}+\tilde{k}_{j+2}\right) e^{\left[i k_{j+1}\left(x_{j+1}-x_{j}\right)\right]}+\left(\tilde{k}_{j}+\tilde{k}_{j+1}\right)\left(\tilde{k}_{j+1}-\tilde{k}_{j+2}\right) e^{\left[-i k_{j+1}\left(x_{j+1}-x_{j}\right)\right]}\right\}, \\
T_{11}^{(j, j+2)}=T_{22}^{(j, j+2) *}, T_{21}^{(j, j+2)}=T_{12}^{(j, j+2) *} .
\end{gathered}
$$

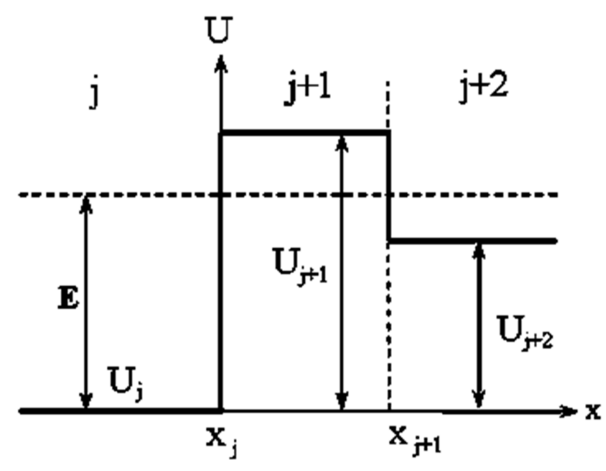

Figure 3. Structure with one potential barrier.

Note here the following:
1. The coefficient $t_{j \rightarrow j+2}$ is invariant to transformation $j \leftrightarrow(j+2)$, which means the coefficient of transmission does not depend on which side electrons go on the potential barrier.

2. The coefficients $t_{j \rightarrow j+2}$ and $r_{j \rightarrow j+2}$ are true for both above-barrier $\left.(E\rangle U_{j}\right)$ and under-barrier $\left(E\left\langle U_{j}\right)\right.$ passage of electrons. In the latter case, it is convenient to use type $\tilde{k}_{m+n}=i \tilde{\kappa}_{m+n}$, $\tilde{k}_{m} \pm \tilde{k}_{m+n}=\sqrt{\left(\tilde{k}_{m}\right)^{2} \pm\left(\tilde{\kappa}_{m+n}\right)^{2}} e^{ \pm i \phi_{m, m+n}} \quad$ transformations when, $\tilde{k}_{m}$ is real, and $\tilde{k}_{m+n}$ is imaginary quantity, 
where $\operatorname{arctg}\left(\phi_{m, m+n}\right)=\frac{\tilde{\kappa}_{m+n}}{\tilde{k}_{m}}$. Then, it should be noted that during the transition from one area to another in waves electrons there should be a phase shift, which is

$$
t_{j \rightarrow j+2}=4\left\{\left(1+\frac{m_{j}}{m_{j+2}}\right)^{2}+\left[1-\left(\frac{k_{j} m_{j+1}}{k_{j+1} m_{j}}\right)^{2}\right]\left[1-\left(\frac{k_{j+1}}{k_{j}} \frac{m_{j}}{m_{j+1}}\right)^{2}\right] \sin ^{2}\left[k_{j+1}\left(x_{j+1}-x_{j}\right)\right]\right\}^{-1} .
$$

4. In the asymmetric (and in the symmetric, but with different effective masses of electrons in different regions (layers)) structure should be observed oscillation in the spectral dependence as a coefficient $t_{j \rightarrow j+2}$, i.e. in the effect of tunneling, and in the transparency coefficient of the potential barrier. The amplitude of this oscillation is determined by the difference between the wave vectors of electrons located in the potential barrier and in the neighboring related not to the coincidence of the phases of the waves propagating in different, but in neighboring, areas.

3. For a symmetric structure with $U_{j}=U_{j+2}$ we have

$$
t^{(j, j+2)}=16 \frac{\tilde{k}_{j+2}}{\tilde{k}_{j}} \frac{1}{\left|\left(1+\frac{\tilde{k}_{j+1}}{\tilde{k}_{j}}\right)\left(1+\frac{\tilde{k}_{j+2}}{\tilde{k}_{j+1}}\right)+\left(1-\frac{\tilde{k}_{j+1}}{\tilde{k}_{j}}\right)\left(1-\frac{\tilde{k}_{j+2}}{\tilde{k}_{j+1}}\right) e^{-2 i k_{j+1}\left(x_{j+1}-x_{j}\right)}\right|^{2}}
$$

It should be noted that both in the above-barrier and in theover-barrier passage of electrons, oscillations of tunneling arise due to the interference of reflected de Broglie waves. The amplitude, in this case, is determined not only by the values of the wave vectors, but on the values of the effective masses of the carriers. Note also that this oscillation does not disappear even in symmetric structures, if they have a difference in the effective masses of current carriers located in two adjacent regions. According to the last expression, as the difference $\left(U_{j} / m_{j}\right)-\left(U_{j+2} / m_{j+2}\right)$ decreases, the oscillation becomes less pronounced and when it disappears, even $\left(U_{j} / m_{j}\right)=\left(U_{j+2} / m_{j+2}\right)$ the structure will be asymmetric.

Figure 4 shows the dependence $t^{(j, j+2)}$ on $\left(x_{j+1}-x_{j}\right)$ for the structure from $A l A s-I n_{0.53} G a_{0.47} A s$ - InAs, from which it can be seen that the oscillation frequency increases with increasing electron energy. Where calculating the following parameters are selected: $U_{j}=55 \mathrm{meV} \quad U_{j+1}=35 \mathrm{meV}$ $U_{j+2}=45 \mathrm{meV} \quad, \quad m_{j+1}=0.046 \cdot m_{0}, \quad m_{j+2}=0.023 \cdot m_{0}$, $m_{j+3}=0.124 \cdot m_{0}$. Calculations show that if we increase the effective mass by 1.6 times, then the amplitude and frequency of oscillations increase by about 1.2 times, which means it is can be controled the oscillation by choosing a sample of different chemical composition. potential wells, i.e. $\left(\tilde{k}_{j+1}-\tilde{k}_{j}\right)$ and $\left(\tilde{k}_{j+2}-\tilde{k}_{j+1}\right)$ Such an interference phenomenon in the structure does not disappear even in a symmetric structure due to the difference between the effective masses of electrons in different layers of the structure.

Next, we define the expression for the coefficient of transmission through the structures from region $j$ to region $j$ +2 for an arbitrary ratio of the carrier energy to the height of the potential barrier. So 


$$
\begin{array}{r}
\left(\begin{array}{l}
0 \\
b_{1}
\end{array}\right)=\hat{T}^{(3)}\left(\begin{array}{l}
a_{3} \\
0
\end{array}\right)=\left(\begin{array}{cc}
T_{11}^{(3)} & T_{12}^{(3)} \\
T_{21}^{(3)} & T_{22}^{(3)}
\end{array}\right)=\left(\begin{array}{l}
a_{3} \\
0
\end{array}\right)=\left(\begin{array}{cc}
T_{11}^{(3)} a_{3} \\
T_{21}^{(3)} b_{3}
\end{array}\right),(13) \quad \begin{array}{l}
\text { whence the condition of existence } \\
e^{i\left(k_{3} x_{2}-k_{1} x_{1}\right)}
\end{array}\left[\left(1+\frac{\tilde{k}_{2}}{\tilde{k}_{1}}\right)\left(1+\frac{\tilde{k}_{3}}{\tilde{k}_{2}}\right) e^{-i k_{2}\left(x_{2}-x_{1}\right)}+\left(1-\frac{\tilde{k}_{2}}{\tilde{k}_{1}}\right)\left(1-\frac{\tilde{k}_{3}}{\tilde{k}_{2}}\right) e^{i k_{2}\left(x_{2}-x_{1}\right)}\right]=0
\end{array}
$$

Next, we determine the localization energy of electrons in their both above-barrier and over-barrier transport, where, for simplify of further analysis of the results, we assume that the regions " 1 " and " 3 " are physically same.

From the last, it is not difficult to obtain that the localized level is dimensionally quantized, i.e. $E_{3,1}\left(k_{y}=k_{z}=0 ; n_{3,1}\right)=\frac{\pi^{2} \hbar^{2} n_{3,1}^{2}}{8 m_{3,1}\left(x_{2}-x_{3,1}\right)^{2}}$ and are located in areas " 1 " and " 3 ", where $n_{3,1}=0,1,2, \ldots$. If such dimensional quantization does not occur, then the localization energy in the current case, when the regions " 1 " and " 3 " are physically same, is determined from the following transcendental equations:

for above-barrier transition of electrons

$$
\frac{\left(\tilde{k}_{2}+\tilde{k}_{1}\right)^{2}}{\tilde{k}_{2}^{2}-\tilde{k}_{1}^{2}}=\cos \left[k_{2}\left(x_{2}-x_{1}\right)\right]
$$

for over-barrier transition of electrons

$$
e^{\kappa_{2}\left(x_{2}-x_{1}\right)}=\frac{\tilde{\kappa}_{2}^{2}-\tilde{k}_{1}^{2}}{\tilde{\kappa}_{2}^{2}+\tilde{k}_{1}^{2}} .
$$

whence the condition of existence of localized states looks

From (15) it is easy to obtain that in the structure under consideration there is only one localized level of electrons, regardless of what the thickness of the layers.

For a structure with the same effective electron masses, during the sub-barrier electron transition, dimensional quantization of their local states occurs, which is determined by the expression

$$
E_{n}^{(2)}=\frac{\pi^{2} \hbar^{2}}{8 m_{2}\left(x_{2}-x_{1}\right)^{2}}+U_{2}
$$

\subsection{Resonant Tunneling Through a Double-barrier Structure, Taking Into Account the Bastard Condition}

Consider the over-barrier transition of electrons with energy $E\left\langle U_{j+1, j+3}\right.$ (i.e. $k_{j+1}=i \kappa_{j+1}, k_{j+3}=i \kappa_{j+3}$ ) through an asymmetric structure. Then the matrix element of the transfer matrix (12) in the case $j=1$ is determined by the sum of the multiple $A_{1} \times A_{2}$ and $B_{1} \times B_{2}$, where

$$
\begin{gathered}
A_{1}=e^{i\left(k_{3} x_{2}-k_{1} x_{1}\right)} \xi_{1}, A_{2}=e^{i\left(k_{5} x_{4}-k_{3} x_{3}\right)} \xi_{2}, \\
B_{1}=e^{-i\left(k_{3} x_{2}+k_{1} x_{1}\right)} \xi_{1}^{*}, \quad B_{2}=e^{-i\left(k_{5} x_{4}+k_{3} x_{3}\right)} \xi_{2}^{*},
\end{gathered}
$$

where

$$
\begin{gathered}
\xi_{1}=\frac{i}{4}\left(1+\frac{\tilde{\kappa}_{2}^{2}}{\tilde{k}_{1}^{2}}\right)^{1 / 2}\left(1+\frac{\tilde{k}_{3}^{2}}{\tilde{\kappa}_{2}^{2}}\right)^{1 / 2}\left[e^{\kappa_{2}\left(x_{2}-x_{3}\right)} e^{i\left(\phi_{21}-\phi_{32}\right)}-e^{-\kappa_{2}\left(x_{2}-x_{1}\right)} e^{-i\left(\phi_{21}-\phi_{32}\right)}\right], \\
\xi_{2}=\frac{i}{4}\left(1+\frac{\tilde{\kappa}_{4}^{2}}{\tilde{k}_{3}^{2}}\right)^{1 / 2}\left(1+\frac{\tilde{k}_{5}^{2}}{\tilde{\kappa}_{4}^{2}}\right)^{1 / 2}\left[e^{-\kappa_{4}\left(x_{4}-x_{3}\right)} e^{i\left(\phi_{43}-\phi_{54}\right)}-e^{\kappa_{4}\left(x_{4}-x_{3}\right)} e^{-i\left(\phi_{43}-, \phi_{54}\right)}\right], \phi_{21}=\operatorname{arctg}\left(\tilde{\kappa}_{2} / \tilde{k}_{1}\right), \phi_{32}=\operatorname{arctg}\left(\tilde{k}_{3} / \tilde{\kappa}_{2}\right), \\
\phi_{43}=\operatorname{arctg}\left(\tilde{\kappa}_{4} / \tilde{k}_{3}\right), \phi_{54}=\operatorname{arctg}\left(\tilde{k}_{4} / \tilde{x}_{3}\right)
\end{gathered}
$$

Then we have

$$
T_{11}^{(5)}=\frac{-1}{16} e^{i\left(k_{5} x_{4}-k_{1} x_{1}\right)}\left(1+\frac{\tilde{\kappa}_{2}^{2}}{\tilde{k}_{1}^{2}}\right)^{1 / 2}\left(1+\frac{\tilde{k}_{3}^{2}}{\tilde{\kappa}_{2}^{2}}\right)^{1 / 2}\left(1+\frac{\tilde{\kappa}_{4}^{2}}{\tilde{k}_{3}^{2}}\right)^{1 / 2}\left(1+\frac{\tilde{k}_{5}^{2}}{\tilde{\kappa}_{4}^{2}}\right)^{1 / 2} e^{x_{4}\left(x_{4}+x_{3}\right)} e^{x_{2}\left(x_{2}+x_{1}\right)} \cdot K
$$

where

$$
\begin{aligned}
& K=2 i\left\{e^{-2 \kappa_{2}\left(x_{2}-x_{1}\right)} e^{-2 \kappa_{4}\left(x_{4}-x_{3}\right)} e^{-i\left(\phi_{21}+\phi_{54}\right)} \sin \left[-k_{3}\left(x_{3}-x_{2}\right)-\phi_{32}-\phi_{43}\right]+\right. \\
& +e^{-2 \kappa_{4}\left(x_{4}-x_{3}\right)} e^{i\left(\phi_{21}-\phi_{54}\right)} \sin \left[k_{3}\left(x_{3}-x_{2}\right)-\phi_{32}+\phi_{43}\right]+ \\
& +e^{-2 \kappa_{2}\left(x_{2}-x_{1}\right)} e^{i\left(-\phi_{21}+\phi_{54}\right)} \sin \left[k_{3}\left(x_{3}-x_{2}\right)+\phi_{32}-\phi_{43}\right]+ \\
& \left.+e^{i\left(\phi_{21}+\phi_{54}\right)} \sin \left[-k_{3}\left(x_{3}-x_{2}\right)+\phi_{32}+\phi_{43}\right]\right\}
\end{aligned}
$$

Thus, the expression for the transparency of barriers is

$$
t^{(1,5)}=\frac{16^{2} \tilde{k}_{1} \tilde{\kappa}_{2}^{2} \tilde{k}_{3}^{2} \tilde{\kappa}_{4}^{2} \tilde{k}_{5} e^{-2 \kappa_{2}\left(x_{2}-x_{1}\right)} e^{-2 \kappa_{4}\left(x_{4}-x_{3}\right)}}{\left(\tilde{k}_{1}^{2}+\tilde{\kappa}_{2}^{2}\right)\left(\tilde{\kappa}_{2}^{2}+\tilde{k}_{3}^{2}\right)\left(\tilde{k}_{3}^{2}+\tilde{\kappa}_{4}^{2}\right)\left(\tilde{\kappa}_{4}^{2}+\tilde{k}_{5}^{2}\right)|K|^{2}}
$$

For the completeness, we further consider that the potential energies $U_{1}, U_{3}, U_{5}$ exceed the electron energy $\left.\left(U_{1}, U_{3}, U_{5}\right\rangle E\right)$ and $k_{2}=k_{4}, x_{2}-x_{1}=x_{4}-x_{3}$, then, the energy spectrum of electrons in a structure with a potential 
well divided with low transparency, i.e. when $\exp \left[-\kappa_{3}\left(x_{3}-x_{2}\right)\right] \ll<1$ described by the expression $E^{(0)}=\frac{\hbar^{2} k_{2}^{(0)^{2}}}{2 m_{2}}+U_{2}$, where $k_{2}^{(0)}$ is determined from the condition $\sin \left[\phi_{12}+\phi_{32}-k_{2}^{(0)}\left(x_{2}-x_{1}\right)\right]=0$. Next, we define the energy spectrum of localized states of electrons in a structure with a rectangular asymmetric potential well, where we also assume that the physicochemical properties of the potential wells " 1 ", " 3 ", " 5 " and barriers " 2 ", " 4 " are the same. In this case, the matrix element of the transfer matrix is

$$
\begin{gathered}
T_{11}^{(5)}=A_{1} \times A_{2}+B_{1} \times B_{2}, \\
T_{11}^{(5)}=\frac{1}{4 \tilde{k}_{1}^{2} \tilde{k}_{2}^{2}}\left\{\left[e ^ { i k _ { 1 } x _ { 2 1 } } \left(2 \tilde{k_{1}} \tilde{k_{2}} \cos \left(k_{2} x_{21}\right)\right.\right.\right. \\
T_{1}^{(5)}=\frac{1}{4 \widetilde{k}_{1}^{2} \widetilde{k}_{2}^{2}}\left\{\left[\left(\widetilde{k}_{2}^{2}+\widetilde{k}_{1}^{2}\right) \sinh \left(k_{2} x_{21}\right)\right]^{2}-\right. \\
\left.-\left[e^{i k_{1} x_{21}}\left(\left(\widetilde{k}_{1}^{2}-\widetilde{k}_{2}^{2}\right) \sinh \left(k_{2} x_{21}\right)+2 \widetilde{k}_{1} \widetilde{k}_{2} \cosh \left(k_{2} x_{21}\right)\right)\right]^{2}\right\} .
\end{gathered}
$$$$
T_{11}^{(5)}=\frac{1}{4 \tilde{k}_{1}^{2} \tilde{k}_{2}^{2}}\left\{\left[e^{i k_{1} x_{21}}\left(2 \tilde{k}_{1} \tilde{k}_{2} \cos \left(k_{2} x_{21}\right)-i\left(\tilde{k}_{1}^{2}+\tilde{k}_{2}^{2}\right) \sin \left(k_{2} x_{21}\right)\right)\right]^{2}-\left[\left(\tilde{k}_{2}^{2}-\tilde{k}_{1}^{2}\right) \sin \left(k_{2} x_{21}\right)\right]^{2}\right\}, ;
$$

Then for localized states of electrons in which energy is greater than the heights of the potential barriers, we have the following transcendental equation

$$
\frac{2 \widetilde{k_{1}} \widetilde{k}_{2}}{\left(\widetilde{k}_{1}^{2}+\widetilde{k}_{2}^{2}\right)}=\operatorname{ctg}\left(k_{1} x_{21}\right) \cdot \operatorname{tg}\left(k_{2} x_{21}\right)
$$

\subsection{Wave Function of Electrons in an Asymmetric Structure}

Consider the wave function for the asymmetric structure shown in Figure $5 \psi_{j}(x)=A_{j} e^{-i k_{j} x}+B_{j} e^{i k_{j} x}, \quad(\mathrm{j}=1,2)$. If we assume that $\mathrm{j}=2$ the layer is a barrier (that is, we believe that), then from the boundary condition of Bastard we have the form (consider that $A_{1}=0$ )

$$
\left\{\begin{array}{l}
2 A_{2} e^{2 i k_{2} x_{1}}=\left(1-\frac{\kappa_{1}}{i \widetilde{k}_{2}}\right) B_{1} e^{-\kappa_{1} x_{1}} e^{i k_{2} x_{1}}, \quad \begin{array}{l}
\text { (27) } \\
\text { the expression for the coefficient } A_{2} \text { is }
\end{array} \\
2 B_{2} e^{-2 i k_{2} x_{1}}=\left(1+\frac{\kappa_{1}}{i \widetilde{k}_{2}}\right) B_{1} e^{-\kappa_{1} x_{1}} e^{-i k_{2} x_{1}}, \\
\left|A_{2}\right|^{-2}=\left(x_{2}-x_{1}\right)+\frac{\sin \left(k_{2}\left(x_{2}-x_{1}\right) \cdot\right.}{k_{2}\left(1+\xi^{2}\right)}\left[( 1 - \xi ^ { 2 } ) \operatorname { c o s } \left(k_{2}\left(x_{2}-x_{1}\right)-2 \xi \sin \left(k_{2}\left(x_{2}-x_{1}\right)\right]\right.\right.
\end{array}\right.
$$

where

$$
\begin{array}{r}
A_{1}=\frac{e^{i k_{1} x_{21}}}{4 \tilde{k}_{1} \tilde{k}_{2}}\left[\left(\tilde{k}_{1}+\tilde{k}_{2}\right)^{2} e^{-i k_{2} x_{21}}-\left(\tilde{k}_{1}-\tilde{k}_{2}\right)^{2} e^{i k_{2} x_{21}}\right], \\
A_{2}=A_{1}\left(x_{21} \rightarrow x_{43}\right), \\
B_{1}=\frac{\left(\tilde{k}_{2}^{2}-\tilde{k}_{1}^{2}\right)}{4 \tilde{k}_{1} \tilde{k}_{2}} e^{-i k_{1}\left(x_{2}+x_{1}\right) 1 / 2}\left(e^{-i k_{2} x_{21}}-e^{i k_{2} x_{21}}\right), \\
B_{2}=B_{1}^{*}\left(x_{21} \rightarrow x_{43}\right), x_{a b}=x_{a}-x_{b} .
\end{array}
$$

For above-barrier electron transport, we have where $\kappa_{1}^{2}=2 m_{1}\left(U_{1}-E\right) / \hbar^{2}$. Then

$$
\psi_{2}(x)=\frac{2 A_{2} e^{i k_{2} x_{1}}}{1+i \xi}\left[\cos \left(k_{2}\left(x-x_{1}\right)-\xi \sin \left(k_{2}\left(x-x_{1}\right)\right)\right] .\right.
$$

where $\xi=\sqrt{\frac{m_{2}\left(U_{1}-E\right)}{m_{1} E}}$.

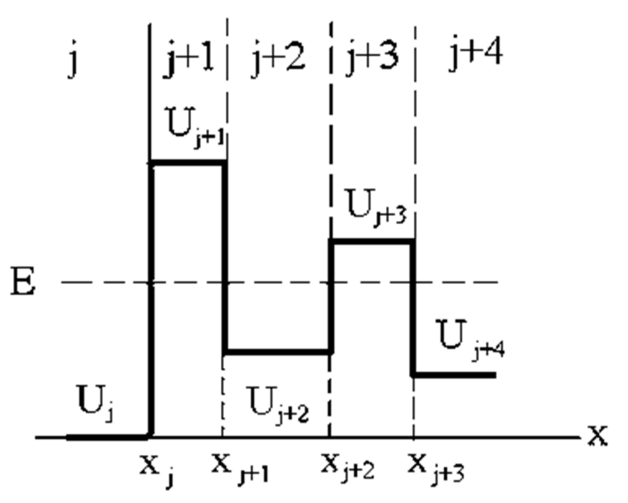

Figure 5. Asymmetric structure with two potential barriers.

From the normalization condition for the wave function, the expression for the coefficient $A_{2}$ is determined by the

\section{Results and Discussion}

We begin the discussion of the obtained results with the expression for $T_{11}^{(j, j+4)}$, where we assume that $j+1, j+3$ the regions are potential barriers (the other potential wells). Then it is not difficult to make sure that even in nanostructures, where potential wells are dimensionally quantized, interference tunneling phenomena can be observed. Note that in this case the degree of observation of 
the interference pattern is described, i.e. controlled only with barrier parameters. Such a phenomenon disappears in the case of a subbarrier transition of electrons when $\left.\left.k_{j+2}\left(x_{j+2}-x_{j+1}\right)\right\rangle\right\rangle 1$ (or $k_{j+2}\left(x_{j+2}-x_{j+1}\right)\langle\langle 1)$. In thes

case

$$
t_{j, j+4}=\frac{k_{j+4}}{k_{j}} \frac{1}{\left|T_{11}^{j, j+2}\right|^{2}}
$$

where

$$
\left|T_{11}^{(j, j+4)}\right|^{2}=\left(4 \widetilde{k}_{j} \widetilde{k}_{j+1} \widetilde{\kappa}_{j+2} \widetilde{k}_{j+3}\right)^{-2} e^{--2 \kappa_{j+2}\left(x_{j+2}-x_{j+1}\right)}\left[\widetilde{k}_{j+1} \widetilde{k}_{j+3}\left(\widetilde{k}_{j}-\widetilde{\kappa}_{j+2}\right)\left(\widetilde{\kappa}_{j+2}-\widetilde{k}_{j+4}\right)\right]^{2}
$$

if conditions are satisfied $k_{j+1}\left(x_{j+1}-x_{j}\right)=\pi n_{j+1}, \quad k_{j+3}\left(x_{j+3}-x_{j+2}\right)=\pi n_{j+3}$ and

$$
\left|T_{11}^{(j, j+4)}\right|^{2}=\left(4 \widetilde{k}_{j} \widetilde{k}_{j+1} \widetilde{\kappa}_{j+2} \widetilde{k}_{j+3}\right)^{-2} e^{--2 \kappa_{j+2}\left(x_{j+2}-x_{j+1}\right)}\left[\left(\tilde{k}_{j+1}^{2}-\widetilde{k}_{j} \widetilde{\kappa}_{j+2}\right)\left(\tilde{k}_{j+3}^{2}-\widetilde{\kappa}_{j+2} \widetilde{k}_{j+4}\right)\right]^{2}
$$

if only conditions are satisfied

$$
k_{j+1}\left(x_{j+1}-x_{j}\right)=\frac{\pi}{2}\left(2 n_{j+1}+1\right), k_{j+3}\left(x_{j+3}-x_{j+2}\right)=\frac{\pi}{2}\left(2 n_{j+3}+1\right)
$$

So in symmetric structures, an oscillation of the coefficient of over-barrier passage of a particle depending on its energy should be observed without taking into account the Bastard condition. Calculations show that with equal values of the width of the well and potential barrier, as well as jumps of the potential of the barrier or well, the amplitude of the oscillations of the over barrier coefficient of passage of particles is greater than the coefficient of passage over the well.

In the case of an asymmetric structure, these arguments remain valid, but the physical nature of the parameters, for example, the number of oscillations, reflection and transmission coefficients, strongly depends on the ratio of the effective masses of electrons in adjacent layers and on the ratio of the height of the left and right potential barrier (in relation to the well).

Note that in an asymmetric (and symmetric, but with different effective masses of electrons in different layers) semiconductor structure, an oscillation should be observed in the dependence of the transmission coefficient through the potential barrier on the electron energy. This oscillation is due to the interference of the waves going to the barrier and reflected from the potential barrier. Such an interference phenomenon in the structure does not disappear even in a symmetric structure due to the difference between the effective masses of electrons located in different regions of the structure.

In the case of an asymmetric structure, these considerations remain valid, but the physical nature of the parameters, for example, the number of oscillations, reflection and transmission coefficients, strongly depends on the ratio of the effective masses of electrons in neighboring layers and on the ratio of the height of the left and right potential barriers (with respect to the well). We note that in an asymmetric (and symmetric, but with different effective masses of electrons in different layers) semiconductor structure, oscillation should be observed depending on the coefficient of transmission through the potential barrier on the electron energy.
This oscillation is due to the interference of waves traveling to the barrier and reflected from the potential barrier. Such an interference phenomenon in the structure does not disappear even in a symmetric structure due to the difference in the effective masses of electrons located in different regions of the structure.

\section{Conclusion}

To calculate a number of kinetic parameters (for example, to calculate the stationary or unsteady conductivity, or the current-voltage characteristic-dependence of the tunneling current on voltage) of asymmetric double-barrier resonancetunneling structures with rectangular barriers, one can use the analytical solution of the Schrödinger equation taking into account the Bastard condition, i.e. taking into account the difference in the effective masses of electrons in neighboring layers of the structure, proposed above. In this case, these kinetic parameters, in particular, the tunneling current that flows through the structure barriers, will depend not only on the parameters of potential barriers and wells, but also on the effective masses of the current carriers. This case requires a separate consideration, to which the next work will be devoted.

\section{Acknowledgements}

This work was partially funded by grant OT-Ф2-66.

\section{References}

[1] A. A. Shchuka. Nanoelectronics. -M., Fizmatkniga. 2007. -465 p.

[2] G. M. Mladenov, V. M. Spivak, E. G. Koleva, A. V. Bogdan. Nanoelectronics. Introduction to nanoelectronic technology. Kiev-Sofia. 1 book. Technosphere. 2009. -327 p.

[3] D. A. Usanov, A. V. Skripal. Physical foundations of nanoelectronics. -Saratov. 2013. $-128 \mathrm{p}$.

[4] V. R. Rasulov. To the Theory of Electron Passage in a Semiconductor Structure Consisting of Alternating Asymmetric Rectangular Potential Wells and Barriers. Russian Physics Journal (Russ Phys J), vol. 59, no. 10, p. 1699-1702, 2017. 
[5] G. Bastard, Wave Mechanics Applied to Semiconductor Heterostructure, Editions de Physique. -Les Ulis, France. 1988. $-317 \mathrm{p}$.

[6] E. L. Ivchenko, G. E. Picus. Superlattices and Other Heterostructures: Symmetry and Optical Phenomena, Springer Series in Solid-State Sciences. -Springer-Verlag, Berlin, Heidelberg. 1995; second edition 1997. -657 p.

[7] I. V. Belyaev, E. I. Golant, A. B. Pashkovsky. Particular properties of resonant interaction of electrons with a highfrequency electric field in two-barrier structures Fizika $i$ texnika poluprovodnilov (FTP) (in russian), vol. 31, no 2, p. 137-144, 1997.

[8] E. I. Golant, A. B. Pashkovsky. A resonant interaction between electrons and high-frequency electric fields in asymmetric double-barrier heterostructures. Fizika $i$ texnika poluprovodnilov (FTP) (in russian), vol. 31, no 9, p. 10771082, 1997.

[9] E. I. Golant, A. B. Pashkovsky. Resonant transitions between splitting energy levels of triple-barrier nanostructures and their application perspectives in submillimeter-wave devices. Fizika i texnika poluprovodnilov (FTP) (in russian), vol. 36, no 3, p. 334-337, 2002

[10] E. I. Golant, A. B. Pashkovsky. Two level electron wave functions in the double-barrier quantum-size structures in a finite amplitude electric field. Fizika i texnika poluprovodnilov (FTP) (in russian), vol. 34, no 3, p. 330-337, 2002.

[11] A. B. Pashkovsky. Resonant transmission of electrons through a three-barrier structure in the two-frequency electric field. Fizika i texnika poluprovodnilov (FTP) (in russian), vol. 45, no 6, p. 759-764, 2011.

[12] V. F. Elesin. High-frequency response of double-barrier nanostructures/ Journal of Experimental and Theoretical Physics (JETP) (in russian), vol. 121, no. 4, p. 925-932, 2002.

[13] V. F. Elesin. Resonance tunneling of electrons interacting with phones. I Journal of Experimental and Theoretical Physics (JETP) (in russian), vol. 123, no. 5, p. 1096-1105, 2003.

[14] V. F. Elesin. Resonant tunneling and nonlinear response in a high frequency field. Journal of Experimental and Theoretical Physics (JETP) (in russian), vol. 124, no. 2, p. 379-393, 2003. -2003. -T. 124. -№2. -C. 379-393.

[15] V. F. Elesin, I. Yu. Kateev. High-frequency properties of double-well nanostructures. Fizika i texnika poluprovodnilov (FTP) (in russian), vol. 42, no 5, p. 586-591, 2008.

[16] V. F. Elesin. High-frequency properties of double-well nanostructures. Journal of Experimental and Theoretical Physics (JETP) (in russian), vol. 145, no. 6, p. 1078-1086, 2014.

[17] V. I. Galiev, A. N. Kruglov, A. F. Polupanov, E. M. Goldis, T. L. Tansley. Multichannel scattering of charge carriers on quantum-well heterostructures. Fizika $i$ texnika poluprovodnilov (FTP) (in russian), vol. 36, no 5, p. 576-581, 2002.

[18] Davies J. N. The Physics of low-dimensional semiconductors. -Cambridge University. -1998. 438 p. 